\title{
Fairness Towards Groups of Agents in the Allocation of Indivisible Items
}

\author{
Nawal Benabbou $^{1}$, Mithun Chakraborty ${ }^{2}$, Edith Elkind ${ }^{3}$, Yair Zick ${ }^{2}$ \\ ${ }^{1}$ Sorbonne Université, CNRS, Laboratoire d'Informatique de Paris 6, LIP6 F-75005 Paris, France \\ ${ }^{2}$ Department of Computer Science, National University of Singapore, Singapore \\ ${ }^{3}$ Department of Computer Science, University of Oxford, United Kingdom \\ nawal.benabbou@lip6.fr, \{mithun, zick\}@comp.nus.edu.sg, elkind@cs.ox.ac.uk
}

\begin{abstract}
In this paper, we study the problem of matching a set of items to a set of agents partitioned into types so as to balance fairness towards the types against overall utility/efficiency. We extend multiple desirable properties of indivisible goods allocation to our model and investigate the possibility and hardness of achieving combinations of these properties, e.g. we prove that maximizing utilitarian social welfare under constraints of typewise envy-freeness up to one item (TEF1) is computationally intractable. We also define a new concept of waste for this setting, show experimentally that augmenting an existing algorithm with a marginal utility maximization heuristic can produce a TEF1 solution with reduced waste, and also provide a polynomial-time algorithm for computing a non-wasteful TEF1 allocation for binary agent-item utilities.
\end{abstract}

\section{Introduction}

Consider an academic department looking to assign incoming graduate students to advisers. It has computed a score for each potential advisor-advisee pair and cares not just about the overall score of the matching but also about a fair distribution of scores across the research clusters into which the faculty is divided. A similar trade-off between efficiency/welfare and fairness might be desirable in other planning/allocation scenarios such as public housing allocation; e.g. the Singapore Housing and Development Board imposes ethnicity quotas on the matching of tenant households to flats in its housing blocks to promote diversity [Benabbou et al., 2018] but an alternative approach could be to aim for a fair distribution of overall utility across ethnic groups.

We can model these problems as a variant of fair allocation of indivisible goods (see e.g. [Bouveret et al., 2016]): We have an underlying weighted bipartite matching problem, the nodes on the two sides corresponding to items and agents; however, the parties we are trying to be fair towards are not individual agents but subsets forming a partition over agents - we call these subsets types. An important aspect of this problem is that all agents in a type do not derive utility from all items in the bundle allocated to that type (unlike the public goods scenario [Fain et al., 2016]), each agent being assigned at most one item. Hence, the parties (types) under consideration end up violating the additive bundle-valuation assumption present in much of recent work. There are some approaches (e.g. [Lipton et al., 2004]) that achieve good fairness guarantees under mild assumptions on the (non-negative, non-decreasing) valuation function but, for that very reason, can result in allocations that are wasteful/inefficient in some way given the structure of our problem, if used naïvely. These considerations necessitate novel solution concepts and techniques for our setting.

\subsection{Our Contributions}

We describe a new model of typewise fair allocation and define our desirable properties in Section 2 - in particular, non-wastefulness and typewise envy-freeness up to one item (TEF1) as well as a marginal envy-based variant of the latter. In Sections 3 and 4, we explore the problems of determining Pareto optimal, TMEF1 allocations and TEF1 allocations that maximize overall sum of weights/utilties respectively. In Section 5, we show experimentally that the classic algorithm of Lipton et al. [2004] equipped with a simple heuristic can produce TEF1 allocations with significantly reduced waste. Section 6 details a polynomial-time algorithm for computing a non-wasteful TEF1 allocation for binary agent-item utilities. We conclude with directions for future research in Section 7.

\subsection{Related Work}

There is a rich body of work on approaches towards the fair allocation of indivisible goods [Bouveret et al., 2016; Markakis, 2017]. A popular fairness concept is envy-freeness [Foley, 1967]. A complete envy-free (EF) allocation may not exist but a relaxation that always does is one that is envy-free up to one item (EF1) [Budish, 2011] where any envy towards an agent can be eliminated by removing an item from its bundle. The bounded-envy, polynomial-time algorithm due to Lipton et al. [2004] also produces an EF1 allocation for general valuation functions [Plaut and Roughgarden, 2018].

Extensions of envy-freeness to groups include strict envyfreeness [Zhou, 1992]; coalition fairness or group envyfreeness [Lahaie and Parkes, 2009] as well as envy-freeness of an individual/a group towards a group [Todo et al., 2011], both under monetary transfers; group fairness as defined 
by Conitzer et al. [2019]. Barman et al. [2018a] recently defined a groupwise extension to the maximin sharebased fairness concept. A major difference of these contributions with our model is that they deal with a $\sigma$-algebra of subsets of agents rather than an exogenously defined partition over agents. Notable papers that define fairness with respect to pre-defined groups of multiple players include Manurangsi and Suksompong [2017], Suksompong [2018], Segal-Halevi and Suksompong [2018], and Elzayn et al. [2019] whose concepts of utility and/or fairness are significantly different from ours. Recent work [Fain et al., 2016; Fain et al., 2018] has also explored non-envy-based fairness criteria in public goods allocation under additive valuations.

We must also mention the literature on statistical fairness (also called group fairness) in the fundamentally different problem domain of classification in machine learning: the equalization of some statistical property of the classifier across groups of data instances based on sensitive/protected attributes ([Dwork et al., 2012; Hardt et al., 2016; Kearns et $a l ., 2018]$ and references therein); we, on the other hand, are interested in fairness notions in terms of subjective valuations of items from the economics/social choice literature.

\section{Model And Definitions}

Throughout the paper, $[r]$ will denote the set $\{1,2, \cdots, r\}$ for any positive integer $r$. Our model, an extension of the classic framework of matching on a weighted bipartite graph [Lovász and Plummer, 2009], has the following ingredients:

(i) a set $N$ of $n$ vertices called agents partitioned into $k$ types $N_{1}, \ldots, N_{k}$,

(ii) a set $M$ of $m$ vertices called items,

(iii) a weight/utility $u(i, j) \in \mathbb{R}_{+}$for each agent-item edge $(i, j) \in N \times M$, such that for at least one $i \in N$ (resp. at least one $j \in M$ ), there is at least one $j \in M$ (resp. each $i \in N)$ with $u(i, j)>0$.

For any $T \subseteq N$ and any $S \subseteq M$, a $(T, S)$-matching is defined as a subset of the edges $T \times S$ such that every vertex in $T \cup S$ is incident on at most one of the edges or, equivalently, as a binary matrix $X=\left(x_{i j}\right)_{i \in T, j \in S}$ such that for each agent $i$ (resp. item $j$ ), there is at most one item $j$ (resp. agent $i$ ) with $x_{i j}=1$, i.e. each item is assigned to at most one agent and each agent is assigned at most one item. The utilitarian social welfare $\operatorname{USW}(X)$ (or total weight) of a matching $X$ is defined as the sum of the realized utilities of all agents under that matching: $\operatorname{USW}(X) \triangleq \sum_{i \in T} \sum_{j \in S} x_{i j} u(i, j)$. An optimal matching is one that maximizes the corresponding USW.

We are interested in a $(N, M)$-matching that trades off some welfare/efficiency concept against some fairness criterion defined with respect to the agent types. More precisely, for every type $p \in[k]$, we are given a type-value function $v_{p}: 2^{M} \rightarrow \mathbb{R}_{+}$which quantifies some concept of overall welfare derived by $N_{p}$ from some bundle or subset of items $S \subseteq M$ in terms of the weights $u(i, j),(i, j) \in N_{p} \times S$. In this paper, we will use the following specific type-value function for every type:

Definition 1 (Utilitarian type-value function). The utilitarian type-value of any type $p \in[k]$ for any bundle $S \subseteq M$ is defined as the total weight of an optimal $\left(N_{p}, S\right)$-matching:

$$
v_{p}(S) \triangleq \begin{cases}\max _{X \in \mathcal{X}\left(N_{p}, S\right)} \operatorname{USW}(X), & \text { if } S \neq \emptyset ; \\ 0, & \text { otherwise. }\end{cases}
$$

where $\mathcal{X}\left(N_{p}, S\right)$ is the collection of all $\left(N_{p}, S\right)$-matchings.

We will define the marginal utility $\Delta_{p}(S ; j)$ of an item $j \in M$ for a type $p \in[k]$ and a bundle $S \subseteq M$ as:

$$
\Delta_{p}(S ; j) \triangleq \begin{cases}v_{p}(S \cup\{j\})-v_{p}(S), & \text { if } j \notin S ; \\ v_{p}(S)-v_{p}(S \backslash\{j\}), & \text { otherwise. }\end{cases}
$$

Given a type $p$ and a bundle $S$, there can be multiple optimal $\left(N_{p}, S\right)$-matchings with the same type-value but possibly differing in other efficiency and/or fairness properties (see Example 1 at the end of this section) - with this in mind, we define an allocation in our setting as follows:

Definition 2 (Allocation). An allocation $\mathcal{A}$ is a collection of bundles $M_{1}^{\mathcal{A}}, \cdots, M_{k}^{\mathcal{A}}$, such that $M_{1}^{\mathcal{A}} \cup \ldots \cup M_{k}^{\mathcal{A}} \subseteq M$ and $M_{p}^{\mathcal{A}} \cap M_{q}^{\mathcal{A}}=\emptyset$ for all $p, q \in[k]$ with $p \neq q$, along with an optimal matching between each type $N_{p}$ and the corresponding bundle $M_{p}^{\mathcal{A}}$ for all $p \in[k]$, thereby inducing a unique $(N, M)$-matching $X^{\mathcal{A}}=\left(x_{i j}^{\mathcal{A}}\right)_{i \in N, j \in M}$.

We call $M_{p}^{\mathcal{A}}$ the allocated bundle of type $p$ under $\mathcal{A}$ and $M_{0}^{\mathcal{A}}=M \backslash \cup_{p \in[k]} M_{p}^{\mathcal{A}}$ the set of withheld items (we will sometimes drop the superscript $\mathcal{A}$ when there is no ambiguity). Thus, item assignment within a type is socially optimal (by design) and EF1 for individual agents (since each is assigned at most 1 item). Type $p$ envies type $q$ if $v_{p}\left(M_{p}^{\mathcal{A}}\right)<$ $v_{p}\left(M_{q}^{\mathcal{A}}\right) ; p$ envies $q$ up to $\nu$ items, $\nu \in\left[\left|M_{q}^{\mathcal{A}}\right|\right]$, if there is a subset $C \subseteq M_{q}^{\mathcal{A}}$ such that $|C|=\nu$ and $v_{p}\left(M_{p}^{\mathcal{A}}\right) \geq$ $v_{p}\left(M_{q}^{\mathcal{A}} \backslash C\right)$ and, for every subset $C^{\prime} \subseteq M_{q}^{\mathcal{A}}$ with $\left|C^{\prime}\right|<\nu$, $v_{p}\left(M_{p}^{\mathcal{A}}\right)<v_{p}\left(M_{q}^{\mathcal{A}} \backslash C^{\prime}\right)$. We can analogously define the envy of a type for a bundle (up to any number of items).

With these fundamentals in place, we now define the desiderata of an allocation $\mathcal{A}$ that we investigate in this paper. The first three are concerned with efficiency; the rest are extensions of efficiency-agnostic fairness concepts introduced by Budish [2011] and Caragiannis et al. [2016] respectively.

Definition 3 (Type-completeness). $\mathcal{A}$ is type-complete if $\cup_{p \in[k]} M_{p}^{\mathcal{A}}=M$; otherwise it is type-incomplete. ${ }^{1}$

Definition 4 (Waste and non-wastefulness). An item $j \in M$ is said to be wasted by an allocation $\mathcal{A}$ if it has a positive marginal utility for some type $p \in[k]\left(\right.$ i.e. $\left.\Delta_{p}\left(M_{p}^{\mathcal{A}} ; j\right)>0\right)$ but is either withheld (i.e. $j \in M_{0}^{\mathcal{A}}$ ) or belongs to the allocated bundle of some type $q \neq p$ for which it has zero marginal utility (i.e. $j \in M_{q}^{\mathcal{A}}$ and $\Delta_{q}\left(M_{q}^{\mathcal{A}} ; j\right)=0$ ). $\mathcal{A}$ is called nonwasteful if it has no wasted item, and wasteful otherwise.

Definition 5 (Typewise Pareto optimality). Allocation $\mathcal{A}_{1}$ is said to typewise Pareto dominate another allocation $\mathcal{A}_{2}$ if $v_{p}\left(M_{p}^{\mathcal{A}_{1}}\right) \geq v_{p}\left(M_{p}^{\mathcal{A}_{2}}\right)$ for all types $p \in[k]$ and $v_{p}\left(M_{p}^{\mathcal{A}_{1}}\right)>$ $v_{p}\left(M_{p}^{\mathcal{A}_{2}}\right)$ for some type $p \in[k]$. An allocation that is not typewise Pareto dominated by any other allocation is typewise Pareto optimal.

\footnotetext{
${ }^{1}$ Type-completness does not preclude an item $j \in M_{p}^{\mathcal{A}}$ remaining unassigned in the $\left(N_{p}, M_{p}^{\mathcal{A}}\right)$-matching.
} 
Definition 6 (Typewise envy-freeness up to one item). Allocation $\mathcal{A}$ is typewise envy-free up to one item (TEF1) if for any two types $p, q \in[k]$, $p$ either does not envy $q$ or envies $q$ up to one item, i.e. there exists an item $j \in M_{q}^{\mathcal{A}}$ such that $v_{p}\left(M_{p}^{\mathcal{A}}\right) \geq v_{p}\left(M_{q}^{\mathcal{A}} \backslash\{j\}\right)$.

Definition 7 (Typewise marginal envy-freeness up to one item). Allocation $\mathcal{A}$ is typewise marginally envy-free up to one item (TMEF1) if for any $p, q \in[k]$, there is an item $j \in M_{q}^{\mathcal{A}}$ such that $v_{p}\left(M_{p}^{\mathcal{A}}\right) \geq v_{p}\left(M_{p}^{\mathcal{A}} \cup M_{q}^{\mathcal{A}} \backslash\{j\}\right)-v_{p}\left(M_{p}^{\mathcal{A}}\right)$.

We are now ready to formulate and analyze specific problems that approach 'good' allocations in different ways. But first, we provide two problem instances that we will use as running examples in the rest of the paper.

Example 1. The types $N_{1}, N_{2}$ and items $M$ are as shown in Figure 1 (left): $u(i, j)$ is 1 if there is an edge between agent $i$ and item $j$, and 0 otherwise. If bundles $M_{1}=\{1,2,6\}$ and $M_{2}=M \backslash M_{1}$ are allocated to $N_{1}$ and $N_{2}$, there is a unique optimal $\left(N_{2}, M_{2}\right)$-matching with $3,4,5$ assigned to $b_{1}, b_{2}, b_{3}$ respectively; but there are two optimal $\left(N_{1}, M_{1}\right)$-matchings in both of which 2 is assigned to $a_{2}$ : If 1 is assigned to $a_{1}$ and 6 remains unassigned, then we have a wasteful allocation since 6 could be assigned to $b_{4}$ so that $\Delta_{2}\left(M_{2} ; 6\right)=1$; but if 6 is assigned to $a_{1}$ instead, the allocation is non-wasteful since no agent in $N_{2}$ has a positive utility for item 1 .

Example 2. The types and items are as shown in Figure 1 (right): All agents of the same type have the same utility for an item, equal to the integer with which the edge between that type and the item is labeled; no edge indicates zero utility.
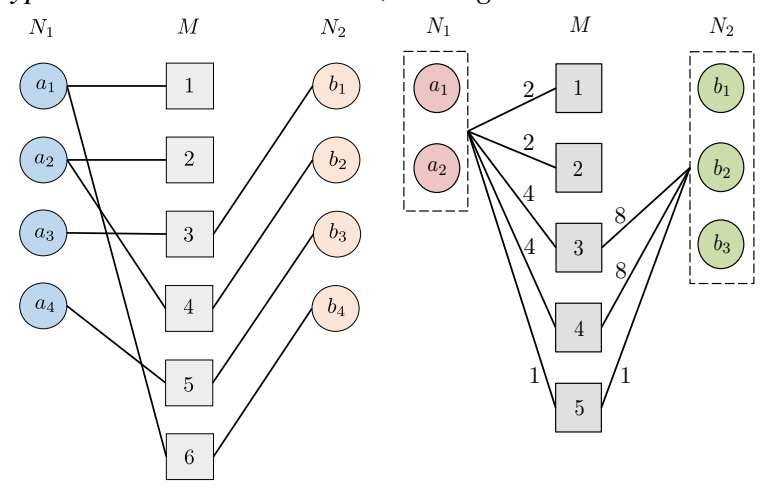

Figure 1: Instances of Examples 1 (left) and 2 (right).

\section{Typewise Pareto Optimal TMEF1 Allocation}

In this section, we will adapt a result of Caragiannis et al. [2016] to our setting. To that end, we first state without proof a property of our type-value function (Definition 1) that is part of the folklore of weighted bipartite matching.

Theorem 1. The utilitarian type-value function $v_{p}(S)$ is a non-additive, monotone submodular function of $S \in 2^{M}$.

Moreover, we define the Nash type-welfare of an allocation $\mathcal{A}$ as the product of the type-values, i.e. $\prod_{p=1}^{k} v_{p}\left(M_{p}^{\mathcal{A}}\right)$.

Theorem 2. Every allocation that maximizes the Nash typewelfare is typewise Pareto optimal and TMEF1.
Proof. Since each valuation function $v_{p}(\cdot)$ is monotone submodular by Theorem 1, a natural extension of Theorem 3.5 of [Caragiannis et al., 2016] suffices to prove the result.

The next result establishes that non-wastefulness is a weakening of typewise Pareto optimality in our setting.

Lemma 1. Any typewise Pareto optimal allocation is nonwasteful but the converse is not true.

Proof. If an allocation $\mathcal{A}$ had a wasted item $j$ with positive marginal utility for a type $q$, then, by the definition of waste, we could augment $M_{q}^{\mathcal{A}}$ with $j$ for an improved type-value without reducing any other type-value, resulting in an allocation that Pareto dominates $\mathcal{A}$. For the converse, Example 1 provides a counterexample: Take again the allocation $\mathcal{A}_{1}$ with bundles $M_{1}^{\mathcal{A}_{1}}=\{1,2,6\}, M_{2}^{\mathcal{A}_{1}}=M \backslash M_{1}^{\mathcal{A}_{1}}$, item 1 remaining unassigned and $2, \ldots, 6$ assigned to $a_{2}, b_{1}, b_{2}, b_{3}, a_{1}$ respectively. This is non-wasteful (and incidentally also TEF1) but is Pareto dominated by allocation $\mathcal{A}_{2}$ with bundles $M_{1}^{\mathcal{A}_{2}}=\{1,2,3\}, M_{2}^{\mathcal{A}_{2}}=M \backslash M_{1}^{\mathcal{A}_{2}}$ since $v_{1}\left(M_{1}^{\mathcal{A}_{2}}\right)=$ $3>2=v_{1}\left(M_{1}^{\mathcal{A}_{1}}\right)$ and $v_{2}\left(M_{2}^{\mathcal{A}_{2}}\right)=v_{2}\left(M_{2}^{\mathcal{A}_{1}}\right)=3$.

This implies, in conjunction with Theorem 2, that a maximum Nash type-welfare allocation is also non-wasteful. Despite the above existence results, maximizing Nash welfare with indivisible items is known to be hard in general, and the above guarantees may break down for constant-factor approximations [Caragiannis et al., 2016]. Moreover, marginal envy-freeness up to one item (MEF1) is a relatively new fairness concept that is less intuitive than envy-freeness up to one item and not extensively used as yet. In fact, it is easy to show that EF1 implies MEF1 for monotone submodular bundle-valuation functions. Hence, we will henceforth focus on efficient TEF1 allocations. Note that an allocation maximizing Nash type-welfare is not necessarily TEF1: In Example 2, any allocation with $M_{1}=\{1,2\}$ and $M_{2}=\{3,4,5\}$ maximizes the Nash type-welfare but is not TEF1 since $v_{1}\left(M_{1}\right)=4$ whereas $v_{1}\left(M_{2} \backslash\{3\}\right)=v_{1}\left(M_{2} \backslash\{4\}\right)=5$, $v_{1}\left(M_{2} \backslash\{5\}\right)=8$.

\section{Assignment under TEF1 Constraints}

We first study the problem of finding a TEF1 allocation $\mathcal{A}$ that maximizes the sum of weights of the induced matching:

$$
\mathrm{USW}\left(X^{\mathcal{A}}\right) \triangleq \sum_{i \in N} \sum_{j \in M} x_{i j}^{\mathcal{A}} u(i, j)=\sum_{p \in[k]} v_{p}\left(M_{p}^{\mathcal{A}}\right) .
$$

This is equivalent to the assignment problem [Munkres, 1957] under TEF1 constraints specified in Definition 6. We define the decision version of the problem as follows:

Definition 8 (ASSIGNTEF1). An instance of the Assignment under TEF1 constraints (ASSIGNTEF1) problem is given by parameters (i) to (iii) of Section 2 as well as a value $U \in \mathbb{R}_{+}$; it is a 'yes'-instance iff it admits a TEF1 allocation $\mathcal{A}$ with a utilitarian social welfare at least $U$.

A TEF1 allocation always exists under our Definition 2 (see Section 5 for further details) but we prove next that it is hard to compute one with the maximum USW for more than 2 types. The hardness question for 2 types is open. 
Theorem 3. The AssignTEF1 problem is NP-complete, even with only 3 types.

Proof. The problem is in NP: given an allocation $\mathcal{A}$, we need to evaluate $k$ type-value functions, solving the polynomialtime unconstrained assignment problem (see e.g. Kuhn [1955]) each time, and can hence verify that $\mathcal{A}$ satisfies all requirements in polynomial time.

We will now describe a polynomial-time reduction to AsSIGNTEF1 from the NP-complete partition problem [Garey and Johnson, 1979]. An instance of the latter is given by a set $S=\left\{s_{j}\right\}_{j \in[l]}$ of $l$ positive integers that sum to $\sigma$; it is a 'yes'-instance iff $S$ can be partitioned into two subsets $S_{1}$ and $S_{2}$ such that both sum to $\sigma / 2$. Given an instance of the partition problem, we construct an ASSIGNTEF1 instance as follows. We have a set of $l+2$ items $M=[l+2]$ and a set of $2 l+4$ agents $N$ partitioned into $k=3$ types $N_{1}=\left\{a_{i}\right\}_{i \in[l+1]}$, $N_{2}=\left\{b_{i}\right\}_{i \in[l+1]}$, and $N_{3}=\left\{c_{1}, c_{2}\right\}$. The utilities are given by $u\left(a_{j}, j\right)=u\left(b_{j}, j\right)=s_{j}, \forall j \in[l] ; u\left(a_{l+1}, j\right)=u\left(b_{l+1}, j\right)=$ $\sigma / 2, \forall j \in\{l+1, l+2\} ; u\left(c_{1}, l+1\right)=u\left(c_{2}, l+2\right)=\kappa$ for an arbitrarily large constant $\kappa>\sigma ; u(i, j)=0$ for every other $(i, j)$-pair. Finally, let $U=2 \kappa+\sigma$.

First, we prove that, for any 'yes'-instance of the partition problem, so is the corresponding ASSIGNTEF1 instance we constructed. Given the two parts $S_{1}$ and $S_{2}$ of $S$ as above, consider the bundles $M_{1}=\left\{j \in M: s_{j} \in S_{1}\right\}, M_{2}=\{j \in$ $\left.M: s_{j} \in S_{2}\right\}$, and $M_{3}=\{l+1, l+2\}$ allocated to $N_{1}$, $N_{2}$, and $N_{3}$ respectively, with no withheld items; evidently, assigning item $j$ to agent $a_{j}$ (resp. $b_{j}$ ) for every $s_{j}$ in $S_{1}$ (resp. $S_{2}$ ), $l+1$ to $c_{1}$, and $l+2$ to $c_{2}$ constitutes the unique optimal matching between each type and its allocated bundle, inducing an allocation $\mathcal{A}$ (Definition 2). We want to prove that $\mathcal{A}$ is TEF1. Note that for the above utilities, $v_{3}\left(M_{3}\right)=$ $2 \kappa>0=v_{3}\left(M_{q}\right)$ for all $q \in\{1,2\} ; v_{1}\left(M_{1}\right)=v_{2}\left(M_{1}\right)=$ $\sum_{s_{j} \in S_{1}} s_{j}=\sigma / 2$ and also $v_{2}\left(M_{2}\right)=v_{1}\left(M_{2}\right)=\sum_{s_{j} \in S_{2}} s_{j}=$ $\sigma / 2$ from the definition of a 'yes'-instance of the partition problem; $v_{1}\left(M_{3} \backslash\{j\}\right)=v_{2}\left(M_{3} \backslash\{j\}\right)=\sigma / 2$ for all $j \in M_{3}$. Hence, each type envies any other type up to at most one item under $\mathcal{A}$. Finally, $\operatorname{USW}\left(X^{\mathcal{A}}\right)=\sigma / 2+\sigma / 2+2 \kappa=2 \kappa+\sigma=U$.

Now let us prove that, assuming our constructed AssignTEF1 instance to be a 'yes'-instance, so is the partition instance. Let $\mathcal{A}$ be an allocation verifying all desiderata. Since $\kappa>\sigma$ and the maximum sum of realized utilities that can be achieved from items $j \in[l]$ is $\sigma$, the only way for $\operatorname{USW}\left(X^{\mathcal{A}}\right)$ to be at least $U=2 \kappa+\sigma$ is to have $\{l+1, l+2\} \subseteq M_{3}^{\mathcal{A}}$ with $c_{1}$ (resp. $c_{2}$ ) assigned to $l+1$ (resp. $l+2$ ). Moreover, since the other items must contribute a sum of realized utilities at least $\sigma$, it is also clear from the utility structure that each $j \in[l]$ must be assigned to either $a_{j}$ or $b_{j}$; hence, $M_{3}=\{l+1, l+2\}$, there are no withheld items, and $v_{1}\left(M_{1}^{\mathcal{A}}\right)+v_{2}\left(M_{2}^{\mathcal{A}}\right)=\sigma$. Now consider the sets $S_{1}=\left\{s_{j} \in S: j \in M_{1}^{\mathcal{A}}\right\}$ and $S_{2}=\left\{s_{j} \in S: j \in M_{2}^{\mathcal{A}}\right\}$ : It is evident that they form a partition of $S$ such that the sum of the values in $S_{1}$ (resp. $S_{2}$ ) equals $v_{1}\left(M_{1}^{\mathcal{A}}\right)$ (resp. $v_{2}\left(M_{2}^{\mathcal{A}}\right)$ ). Since $\mathcal{A}$ is TEF1, we must have $v_{1}\left(M_{1}^{\mathcal{A}}\right) \geq v_{1}\left(M_{3}^{\mathcal{A}} \backslash\{j\}\right)$ for some $j \in M_{3}^{\mathcal{A}}$; but, from our utility structure, $v_{1}\left(M_{3}^{\mathcal{A}} \backslash\{j\}\right)=\sigma / 2$ for all $j \in M_{3}^{\mathcal{A}}$ and so the inequality $v_{1}\left(M_{1}^{\mathcal{A}}\right) \geq \sigma / 2$ holds. Arguing similarly, $v_{2}\left(M_{2}^{\mathcal{A}}\right) \geq \sigma / 2$. But since $v_{1}\left(M_{1}^{\mathcal{A}}\right)+v_{2}\left(M_{1}^{\mathcal{A}}\right)=\sigma$, then $\sum_{s_{j} \in S_{1}} s_{j}=v_{1}\left(M_{1}^{\mathcal{A}}\right)=\sum_{s_{j} \in S_{2}} s_{j}=v_{2}\left(M_{2}^{\mathcal{A}}\right)=\sigma / 2$.

Since $v_{p}(\cdot)$ is a particular submodular function, Theorem 3 implies the following result for the traditional indivisible item allocation setting where each agent receives a bundle.

Corollary 1. For monotone submodular agent valuation functions over bundles, it is NP-hard to compute the EF1 allocation that maximizes the sum of valuations.

One might conjecture that the maximum-USW TEF1 allocation is non-wasteful. But the following surprising result belies this intuition, and raises the question: Does a non-wasteful, TEF1 allocation always exist?

Proposition 1. The TEF1 allocation that maximizes the utilitarian social welfare may waste items, even in a problem instance that admits a non-wasteful TEF1 allocation.

Proof. In Example 2, any allocation with bundles $M_{1}=$ $\{1,2\}$ and $M_{2}=\{3,4\}$, and no other allocation, maximizes USW under TEF1 constraints with USW $=20$. But, such an allocation is wasteful since item 5 is withheld although $\Delta_{2}\left(M_{2} ; 5\right)=1$. However, any allocation with bundles $M_{1}^{\prime}=\{1,3\}$ and $M_{2}^{\prime}=\{2,4,5\}$ is non-wasteful and TEF1 but has USW $=15$.

\section{TEF1 Allocation with Reduced Waste}

In our quest for a TEF1 allocation with no (or, at least, low) waste, we note that it is possible to obtain a type-complete TEF1 allocation in polynomial time by a natural extension (called $L$ hereafter) of the algorithm due to Lipton et al. [2004]: Iterate over the items $j \in M$, giving item $j$ to a type, say $p$, that is currently not envied by any other type for its bundle $M_{p}$; compute an optimal matching with the augmented bundle $M_{p} \cup\{j\}$; construct the envy graph where there is a directed edge from a type $q$ to a type $r$ whenever $q$ envies $r$; eliminate any cycle in this graph by transferring the bundle of every envied type to its envying type on this cycle (to ensure that there is an unenvied type in each iteration), followed by re-matching within each such type. Although no item is withheld, it is possible for the final allocation to be wasteful: An item may be allocated to a type which has zero marginal utility for it or may become unassigned after a bundle is transferred between types.

One heuristic that could reduce waste is to allocate the item to the unenvied type that has the maximum marginal utility for it, breaking further ties uniformly at random, rather than to an arbitrary unenvied type - we call $L$, augmented with this heuristic, $H$. Unfortunately, Example 1 shows that, $H$ can be wasteful in general. Consider the order $1,2, \cdots, 6$ over items: 1 and 2 are obviously allocated to $N_{1}$ while, depending on how ties are broken, $3,4,5$ can all be given to $N_{2}$. With this allocation of item 5 , envy appears for the first time and $N_{1}$ is the only unenvied type. Hence, 6 must go to $N_{1}$, although $\Delta_{1}(\{1,2\} ; 6)=0$, and is wasted. Notice further that if 6 were allocated to $N_{2}$, it would increase $N_{2}$ 's own typevalue but make $N_{1}$ envy $N_{2}$ up to 2 items although $N_{1}$ does not want 6 in conjunction with its current bundle! This is especially disappointing since Example 1 admits three nonwasteful TEF1 allocations, which are also typewise Pareto 
optimal and maximize USW, with bundles $M_{1}^{*}=\{1,2,3\}$, $\{1,2,5\}$, or $\{1,2,3,5\}$, and $M_{2}^{*}=M \backslash M_{1}^{*}$ (each resulting in a unique optimal matching for each type).

Nevertheless, to see how the marginal utility maximization heuristic performs in practice, we experimentally compared procedures $L$ and $H$ using the percentage of items wasted as our performance metric. We simulated two sets of problem instances with $n=100$ agents partitioned into $k=3$ types:

UNEQUAL: $\left|N_{1}\right|=74,\left|N_{2}\right|=13,\left|N_{3}\right|=13 .^{2}$

EQUAL: $\left|N_{p}\right| \approx n / k$ for all types $p \in[k]$.

For each, we used $m \in\{50,100\}$ items; for each agent, we sampled $m$ numbers uniformly at random from $[0,1]$ and normalized them to generate utilities for all $m$ items. We report results averaged over 100 runs each.

For the UNEQUAL set, $39 \%$ (resp. 13\%) of the items are wasted on average for $m=100$ (resp. $m=50$ ) by $L$ whereas no item is wasted by $H$; for the EQUAL set, $L$ wastes only $0.005 \%$ of the items with $m=100$ and $n o$ item with $m=50$, and again $n o$ item is wasted by $H$ for either value of $m$. Thus, we can conclude that the performance of the natural extension of [Lipton et al., 2004] strongly depends on parameters such as type proportions and the number of items, whereas augmenting it with the heuristic under consideration gives surprisingly good results over a variety of input instances.

\section{Binary Utilities: Non-Wasteful TEF1 Allocation}

In this section, we fill focus on the binary utility model: $u(i, j) \in\{0,1\}, \forall i \in N, \forall j \in M$. This captures scenarios where each agent either approves or disapproves of an item but does not distinguish among its approved items. There exists prior work on fair allocation algorithms producing allocations with binary item utilities [Barman et al., 2018b] but most assume additive bundle valuations.

Theorem 4. For any problem instance with a binary utility model, there exists a non-wasteful TEF1 allocation that can be computed in polynomial time.

Our proof is constructive: We provide and analyze an allocation algorithm for the problem (Algorithm 1). Like Lipton et al. [2004], we iterate over the items; however, we augment a type's bundle with an item not based on (the absence of) envy towards it but on the marginal utility of the type for the item. Let us call any algorithm that starts with empty bundles and follows the principle of augmenting the current bundle $M_{p}$ of a type $p$ with an item $j$ only if $p$ has positive marginal utility (i.e. $\Delta_{p}\left(M_{p} ; j\right)>0$ ) a PMU algorithm.

Proposition 2. Under the binary utility model, we have $v_{p}(S) \leq \min \left\{\left|N_{p}\right|,|S|\right\}, \forall p \in[k], \forall S \subseteq M$. In particular, any type's value for its allocated bundle at any stage of a $P M U$ algorithm is equal to the cardinality of the bundle, i.e. $v_{p}\left(M_{p}\right)=\left|M_{p}\right|, \forall p \in[k]$.

\footnotetext{
${ }^{2}$ These numbers roughly follow the proportions of Chinese, Malay, and Indian/Other residents of Singapore according to the 2010 census report [Department of Statistics, Singapore, 2010].
}

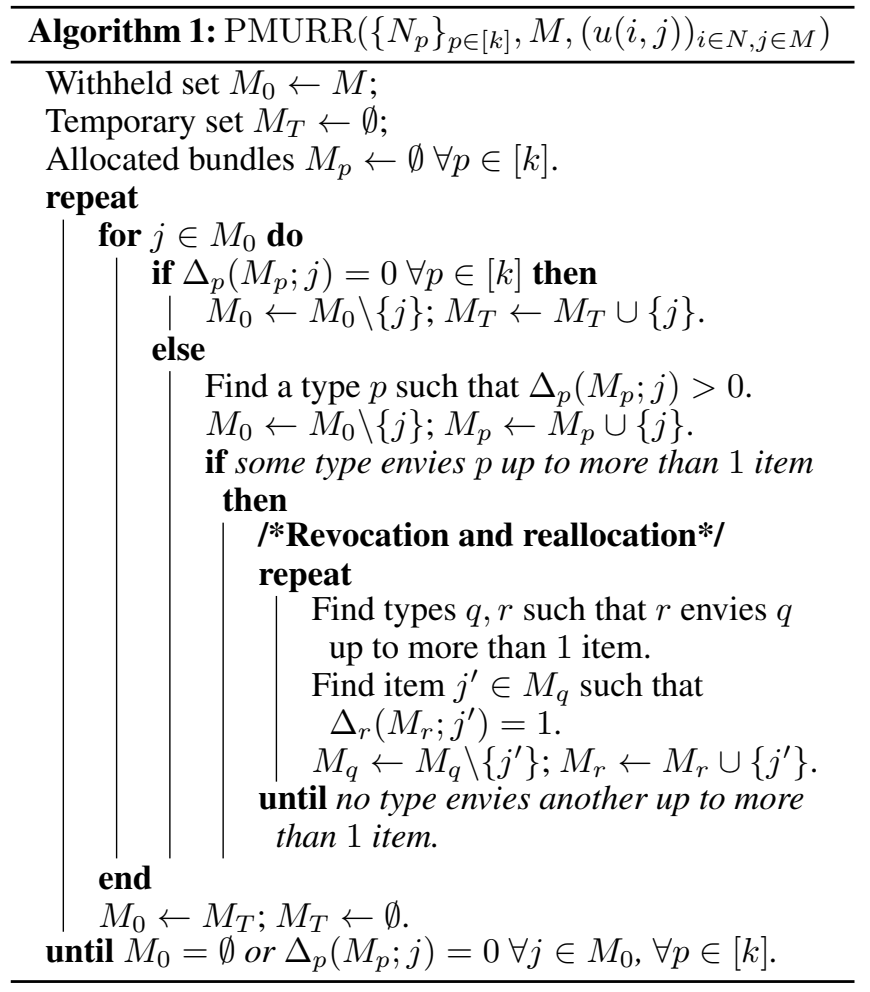

Proof. The first part follows directly from the fact that, for binary utilities, any item can contribute either 1 or 0 to the type-value of a bundle, i.e. $\Delta_{p}(S ; j) \in\{0,1\}$ for any $p \in[k]$, $S \subseteq M$, and $j \notin S$. For the second part, note that each type starts with an empty bundle (hence zero type-value) and increases its type-value by 1 every time it acquires an item under a PMU algorithm; moreover, since all positive utilities are equal, the only way for an item $j$ to improve the type-value of $p$ is to ensure that every item is assigned to an agent in every $\left(N_{p}, M_{p} \cup\{j\}\right)$-matching - hence, if an item is revoked, both the bundle-size and type-value diminish by 1 .

Corollary 2. A type $p \in[k]$ with $\left|M_{p}\right|=\left|N_{p}\right|$ under a PMU algorithm cannot envy any bundle $S \subseteq M$ since $v_{p}(S) \leq\left|N_{p}\right|$.

If after receiving a new item, a type is still envied by all other types up to at most 1 item, no further action is necessary in that iteration. But a PMU approach by itself cannot ensure that no type will start envying the recipient up to more than 1 item if the latter was already envied up to 1 item. If envy does exceed the acceptable limit, we execute a special revocation and reallocation (RR) subroutine repeatedly until we restore the TEF1 property - hence, we call our algorithm PMURR. The functioning of the RR subroutine depends on the following result:

Proposition 3. At any stage of a PMU algorithm, if type $p$ envies type $q$ up to $\nu$ or more items, then $\left|M_{q}\right| \geq\left|M_{p}\right|+\nu$.

Proof. It follows from the definition of envy up to $\nu$ (or more) items: For any subset $C \subseteq M_{q}$ with $|C|=\nu-1, v_{p}\left(M_{p}\right)<$ $v_{p}\left(M_{q} \backslash C\right)$. But, from Proposition 2 (and since $C \subseteq M_{q}$ ), we have $v_{p}\left(M_{q} \backslash C\right) \leq\left|M_{q} \backslash C\right|=\left|M_{q}\right|-|C|=\left|M_{q}\right|-\nu+1$. 
Combining these with Proposition $2\left(v_{p}\left(M_{p}\right)=\left|M_{p}\right|\right)$, we get $\left|M_{p}\right|<\left|M_{q}\right|-\nu+1$, i.e. $\left|M_{p}\right| \leq\left|M_{q}\right|-\nu$.

Corollary 3. Type p can envy another type q only if $q$ has an allocated bundle of a larger size than $p$ which, in turn, implies that there can be no cycles in the envy graph among types for binary utilities under a PMU algorithm.

Next, we prove that the existence of envy is sufficient for having an item that can be revoked and reallocated.

Lemma 2. At any stage of a PMU algorithm, if type p envies type $q$, then there exists an item $j$ in the bundle $M_{q}$ which has a positive marginal utility for $p$, i.e. $\Delta_{p}\left(M_{p} ; j\right)=1$.

Proof. By Proposition 2 and Corollary 2, if $N_{p}^{\prime}$ is the subset of agents who are assigned items in the current $\left(N_{p}, M_{p}\right)$ matching, then we must have $v_{p}\left(M_{p}\right)=\left|M_{p}\right|=\left|N_{p}^{\prime}\right|<$ $\left|N_{p}\right|$ for $p$ to envy $q$. Now, if $N_{p}^{q}$ is the subset of agents who would be assigned items in a $\left(N_{p}, M_{q}\right)$-matching, we must have $\left|N_{p}^{q}\right|=v_{p}\left(M_{q}\right)>v_{p}\left(M_{p}\right)=\left|N_{p}^{\prime}\right|$, so that $\left|N_{p}^{q} \backslash N_{p}^{\prime}\right| \geq$ $\left|N_{p}^{q}\right|-\left|N_{p}^{\prime}\right|>0$. Since $N_{p}^{q} \backslash N_{p}^{\prime} \subseteq N_{p} \backslash N_{p}^{\prime}$, each agent in $N_{p}^{q} \backslash N_{p}^{\prime}$ is assigned no item under the current matching but has a positive utility for a distinct item in $M_{q}$.

A revocation from $q$ (resp. a reallocation to $r$ ) decrements (resp. increments) its type-value, thus reducing the envy of $r$ towards $q$; but it is not obvious how it affects all envy relations (types that already envied $r$ up to 1 or more items, those that $q$ might start envying up to 1 or more items, etc.) and whether we could trigger a never-ending chain reaction. Our final lemma dispels such doubts.

Lemma 3. The revocation and reallocation subroutine produces a TEF1 allocation in a polynomial number of steps.

Proof. First note that computing a type-value for any bundle is polynomial-time and so is checking whether $r$ envies $q$ up to more than 1 item (i.e. $v_{r}\left(M_{r}\right)<v_{q}\left(M_{q} \backslash\{j\}\right)$ for some $j \in M_{q}$ ). Now, for an iteration of the RR subroutine to occur, we must have a type $r$ envying a type $q$ up to 2 or more items, so that $\left|M_{q}\right| \geq\left|M_{r}\right|+2$ by Proposition 3 . For any collection of bundles $\bar{M}=\left\{M_{1}, \ldots M_{2}\right\}$, define the potential function $\Phi(\bar{M}) \triangleq \sum_{p \in[k]}\left|M_{p}\right|^{2}$, and let $\bar{M}^{\prime}=\left\{M_{1}^{\prime}, \ldots M_{2}^{\prime}\right\}$ be the collection of bundles after revocation from $q$ and reallocation to $r$. Hence, $\left|M_{q}^{\prime}\right|=\left|M_{q}\right|-1$ and $\left|M_{r}^{\prime}\right|=\left|M_{r}\right|+1$ (by Proposition 2) but $\left|M_{p}^{\prime}\right|=\left|M_{p}\right|$ for every other type $p$. Thus,

$$
\begin{aligned}
\Phi\left(\bar{M}^{\prime}\right)-\Phi(\bar{M}) & =\left|M_{q}^{\prime}\right|^{2}-\left|M_{q}\right|^{2}+\left|M_{r}^{\prime}\right|^{2}-\left|M_{r}\right|^{2} \\
& =2\left(1+\left|M_{r}\right|-\left|M_{q}\right|\right), \text { on simplification } \\
& \leq 2(1-2)=-2,
\end{aligned}
$$

i.e. $\Phi$ strictly decreases with each RR iteration, and obviously lies between 0 and $m^{2}$ (since $\sum_{p \in[k]}\left|M_{p}\right| \leq m$ ). Hence, RR terminates after a polynomial number of iterations; by the stop criterion, the final allocation is TEF1.

We are now ready to prove the main result of this section.
Proof of Theorem 4. To prove that the final allocation is nonwasteful, note that by construction, no bundle-augmentation and revocation-and-reallocation allow an item in an allocated bundle to remain unassigned; by Corollary 3, no envy cycles are ever formed in the envy graph among types, hence bundles are never passed between types (unlike in the decycling procedure of [Lipton et al., 2004]) and so no item once assigned can become unassigned (or transferred to the temporary set). However, within the "for" loop, the revocation(s) might result in an agent becoming unassigned who has a positive utility for a currently withheld item that was put in the temporary set because it previously had zero marginal utility for each type (hence this item now becomes a wasted item). This necessitates the outer "repeat" loop whose stop criterion ensures that there are no wasted items in the withheld set at the end of the algorithm.

Lemma 3 ensures that the allocation is TEF1 after every iteration of the "for" loop, hence upon termination.

For proving the polynomial time-complexity, recall that computing $v_{p}(\cdot)$ and $\Delta_{p}(\cdot)$, verifying envy up to more than 1 item, and finding an item to revoke and reallocate are all polynomial-time; Lemma 3 ensures that each iteration of the "for" loop takes polynomial time. With each iteration of the outer "repeat" loop, the size of the withheld set, which starts at $m$, strictly decreases (if it does not, it must mean that each withheld item has zero marginal utility for every type - a stop criterion), hence we have a linear number of iterations.

\section{Discussion And Future Work}

We have introduced and investigated typewise fairness and efficiency concepts for the allocation of indivisible goods. The major open question is whether a non-wasteful TEF1 allocation always exists for arbitrary agent-item utilities. Important properties (e.g. the equality of bundle size and type-value) do not carry over from binary to arbitrary utilities, hence extensions of our PMURR algorithm to general utility models remain elusive. An alternative would be to have heuristic approaches such as the one in Section 5 with provable upper bounds on the amount of waste.

We considered one type-value function $v_{p}(\cdot)$ (Definition 1 ) here; the average utilitarian type-value $\hat{v}_{p}(S) \triangleq v_{p}(S) /\left|N_{p}\right|$ is equivalent to $v_{p}(S)$ for all intents and purposes in this paper. More complex functions such as OWA operators [Yager, 1988] or those that address fairness within a type (e.g. [Bouveret and Lang, 2011]) merit further analysis.

Other possible directions for future research include nonenvy-based fairness concepts (egalitarian type-welfare, proportionality, maximin share etc.) as well as strategic implications of typewise fair allocation algorithms.

\section{Acknowledgments}

Chakraborty and Zick were supported by the Singapore MOE grant R-252-000-625-133 and the Singapore NRF Research Fellowship R-252-000-750-733, Benabbou by the ANR project 14-CE24-0007-01-Cocorico-CoDec, and Elkind by the ERC grant 639945 (ACCORD). The authors would like to thank the anonymous reviewers of IJCAI 2019 and the reviewers and attendees of FAMAS 2019 for their feedback. 


\section{References}

[Barman et al., 2018a] Siddharth Barman, Arpita Biswas, Sanath Kumar Krishnamurthy, and Yadati Narahari. Groupwise maximin fair allocation of indivisible goods. In Proc. AAAI, pages 917-924, 2018.

[Barman et al., 2018b] Siddharth Barman, Sanath Kumar Krishnamurthy, and Rohit Vaish. Greedy algorithms for maximizing Nash social welfare. In Proc. AAMAS, pages 7-13, 2018.

[Benabbou et al., 2018] Nawal Benabbou, Mithun Chakraborty, Vinh Ho Xuan, Jakub Sliwinski, and Yair Zick. Diversity constraints in public housing allocation. Proc. AAMAS, pages 973-981, 2018.

[Bouveret and Lang, 2011] Sylvain Bouveret and Jérôme Lang. A general elicitation-free protocol for allocating indivisible goods. In Proc. IJCAI, pages 73-78, 2011.

[Bouveret et al., 2016] Sylvain Bouveret, Yann Chevaleyre, and Nicolas Maudet. Fair allocation of indivisible goods. In Brandt, Felix and Conitzer, Vincent and Endriss, Ulle and Lang, Jérôme and Procaccia, Ariel D., editor, Handbook of Computational Social Choice, chapter 12, pages 284-310. Cambridge University Press, 2016.

[Budish, 2011] Eric Budish. The combinatorial assignment problem: Approximate competitive equilibrium from equal incomes. Journal of Political Economy, 119(6):1061-1103, 2011.

[Caragiannis et al., 2016] Ioannis Caragiannis, David Kurokawa, Hervé Moulin, Ariel D. Procaccia, Nisarg Shah, and Junxing Wang. The unreasonable fairness of maximum Nash welfare. In Proc. EC, pages 305-322. ACM, 2016.

[Conitzer et al., 2019] Vincent Conitzer, Rupert Freeman, Nisarg Shah, and Jennifer Wortman Vaughan. Group fairness for indivisible good allocation. In Proc. AAAI, 2019. To appear.

[Department of Statistics, Singapore, 2010] Department of Statistics, Singapore. Singapore 2010 Census: Key Indicators of the Resident Population, 2010.

[Dwork et al., 2012] Cynthia Dwork, Moritz Hardt, Toniann Pitassi, Omer Reingold, and Richard Zemel. Fairness through awareness. In Proc. ITCS, pages 214-226. ACM, 2012.

[Elzayn et al., 2019] Hadi Elzayn, Shahin Jabbari, Christopher Jung, Michael Kearns, Seth Neel, Aaron Roth, and Zachary Schutzman. Fair algorithms for learning in allocation problems. In Proc. ACM FAT*, 2019. To appear.

[Fain et al., 2016] Brandon Fain, Ashish Goel, and Kamesh Munagala. The core of the participatory budgeting problem. In Proc. WINE, pages 384-399, 2016.

[Fain et al., 2018] Brandon Fain, Kamesh Munagala, and Nisarg Shah. Fair allocation of indivisible public goods. In Proc. EC, pages 575-592, 2018.

[Foley, 1967] Duncan K. Foley. Resource allocation in the public sector. Yale Economic Essays, 7:45-98, 1967.
[Garey and Johnson, 1979] Michael R. Garey and David S. Johnson. Computers and Intractability: A Guide to the Theory of NP-Completeness. W. H. Freeman \& Co., 1979.

[Hardt et al., 2016] Moritz Hardt, Eric Price, Nati Srebro, et al. Equality of opportunity in supervised learning. In Proc. NIPS, pages 3315-3323, 2016.

[Kearns et al., 2018] Michael Kearns, Seth Neel, Aaron Roth, and Zhiwei Steven Wu. Preventing fairness gerrymandering: Auditing and learning for subgroup fairness. In Proc. ICML, pages 2564-2572, 2018.

[Kuhn, 1955] Harold W. Kuhn. The Hungarian method for the assignment problem. Naval Research Logistics (NRL), 2(1-2):83-97, 1955.

[Lahaie and Parkes, 2009] Sébastien Lahaie and David C Parkes. Fair package assignment. In Proc. AMMA, page 92, 2009.

[Lipton et al., 2004] Richard J. Lipton, Evangelos Markakis, Elchanan Mossel, and Amin Saberi. On approximately fair allocations of indivisible goods. In Proc. EC, pages 125-131. ACM, 2004.

[Lovász and Plummer, 2009] László Lovász and Michael D. Plummer. Matching theory, volume 367. American Mathematical Society, 2009.

[Manurangsi and Suksompong, 2017] Pasin Manurangsi and Warut Suksompong. Asymptotic existence of fair divisions for groups. Mathematical Social Sciences, 89:100 108, 2017.

[Markakis, 2017] Evangelos Markakis. Approximation algorithms and hardness results for fair division with indivisible goods. In Trends in Computational Social Choice, chapter 12, pages 231-247. AI Access, 2017.

[Munkres, 1957] James Munkres. Algorithms for the assignment and transportation problems. Journal of SIAM, 5(1):32-38, 1957.

[Plaut and Roughgarden, 2018] Benjamin Plaut and Tim Roughgarden. Almost envy-freeness with general valuations. In Proc. SODA, pages 2584-2603. SIAM, 2018.

[Segal-Halevi and Suksompong, 2018] Erel Segal-Halevi and Warut Suksompong. Democratic fair allocation of indivisible goods. In Proc. IJCAI, pages 482-488, 2018.

[Suksompong, 2018] Warut Suksompong. Approximate maximin shares for groups of agents. Mathematical Social Sciences, 92:40-47, 2018.

[Todo et al., 2011] Taiki Todo, Runcong Li, Xuemei Hu, Takayuki Mouri, Atsushi Iwasaki, and Makoto Yokoo. Generalizing envy-freeness toward group of agents. In Proc. IJCAI, pages 386-392, 2011.

[Yager, 1988] Ronald R. Yager. On Ordered Weighted Averaging aggregation operators in multicriteria decision making. IEEE Transactions on Systems, Man, and Cybernetics, 18(1):183-190, 1988.

[Zhou, 1992] Lin Zhou. Strictly fair allocations in large exchange economies. Journal of Economic Theory, 57(1):158 - 175, 1992. 